\title{
High-fidelity photometry and astrometry of high-contrast imaged companions using LOCI processing.
}

\author{
Jérôme Maire ${ }^{1}$, Jonathan Gagné ${ }^{2}$, David Lafrenière ${ }^{2}$, James R. \\ Graham $^{3}$ and René Doyon ${ }^{2}$ \\ ${ }^{1}$ Dunlap Institute for Astronomy and Astrophysics, University of Toronto, 50 St. George \\ Street, Toronto, Ontario. M5S 3H4, Canada \\ email: maire@dunlap.utoronto.ca \\ ${ }^{2}$ Université de Montréal, Département de physique C.P.6128, Succ. Centre-Ville H3C 3J7, \\ Montréal, Canada \\ ${ }^{3}$ Astronomy Department, University of California, Berkeley, CA 94720
}

Résumé. Direct imaging and spectroscopy of exoplanets is a key element for understanding planet formation and migration. Such direct detections and characterizations remains technologically challenging, since a very high contrast ratio and small angular separation are involved, and futhermore speckle noise limits the high-contrast imaging performance. We further discuss a speckle subtraction and suppression technique that fully takes advantage of spectral and timedomain information on quasi-static speckles to measure the highest-fidelity photometry as well as accurate astrometry of detected companions.

Keywords. techniques : image processing, techniques : high angular resolution, instrumentation : spectrographs

\section{Introduction}

We propose an extension of the LOCI speckle-suppression algorithm, that simultaneously reduces the contribution of speckles in the reduced images and delivers accurate photometry and astrometry of the companions. This method preserves the companion signal by including its presence in images during the speckle noise suppression. First, it preserves companion brightness during the speckle suppression and delivers accurate photometry and astrometry without any aperture-dependent photometry on residual images. The proposed method is also very fast in terms of computing time as it needs only a single optimization in a limited region. Furthermore, this method is generalized to spectral datacube as delivered by an IFS, taking advantage of both ADI and SDI processing, giving powerful speckle suppression.

Spectral Differential Imaging (SDI, Marois et al. 2000) or Angular Differential Imaging (ADI) algorithm like Locally Optimized Combination of Images (LOCI, Lafrenière et al. 2007) are very efficient for suppressing the speckle noise in high-contrast images, improving the effective contrast ratio and companion detectability. However, the resultant speckle suppression is not ideal for high-fidelity companion photometry, as the signal of the companion itself is affected by these methods which focus on the speckle noise suppression.

\section{Data processing}

The LOCI-PSF method (Maire et al. 2012) have been tested by using simulated data in which we injected fake planets of known brightness. We applied the LOCI-PSF algorithm 


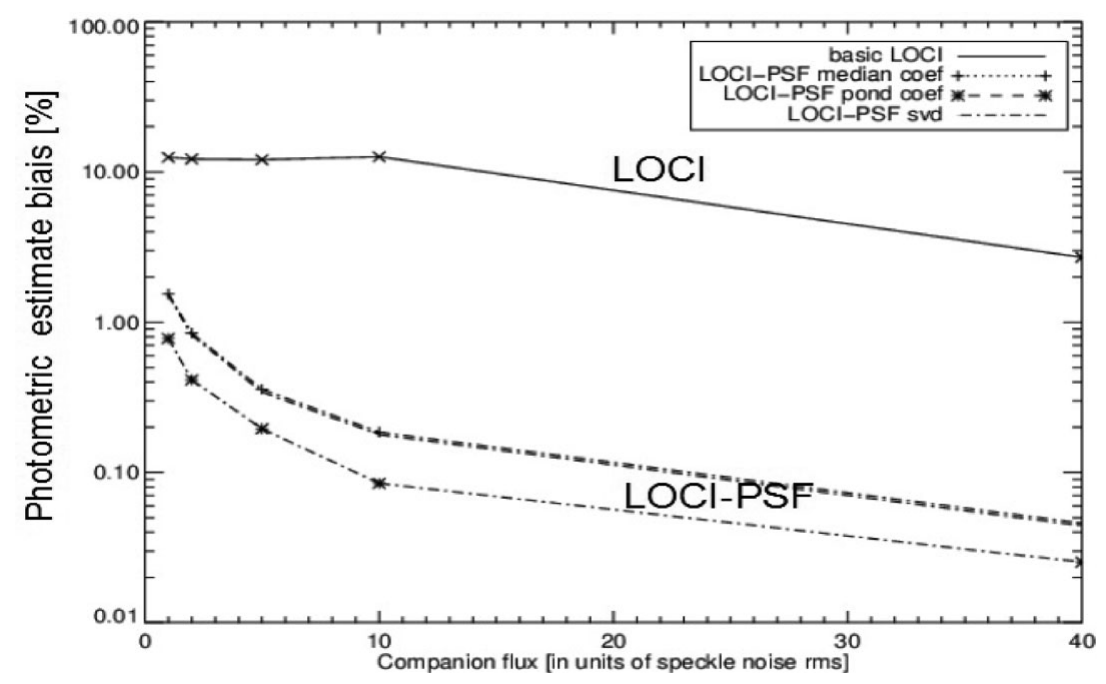

Figure 1. Relative difference between injected and measured companion brightness as a function of companion brightness (temporal sequence of 40 ADI images, with photon noise and varying speckle pattern).

to measure the companion brightness which is compared to the injected one. We placed all our simulations in the case of the Gemini Planet Imager (GPI) at Gemini South Observatory.

GPI coronagraphic AO-corrected H-band PSFs are generated through simulations of the AO control loop, woofer-tweeter correction, and Fresnel wavefront propagation through a high-fidelity optical model of GPI to properly simulate out-of-pupil-plane and finite size optics and model long-lived semi-static speckles produced by internal wavefront aberrations (Poyneer et al. 2006, Poyneer et al. 2007, Véran et al. 1997, Jolissaint et al. 2004).

Results from these simulations show that the LOCI-PSF method produces smaller biais in photometric and astrometric measurement than for standard post-LOCI photometry (Fig. 1). As for standard LOCI algorithms, the photometry given by the LOCI-PSF method is still affected by the variability of the speckle pattern, but at a lower level. Confidence interval can be used to indicate the reliability of the LOCI-PSF companion brightness estimate.

A powerful combination the ADI and LOCI methods into a single one, the Spectral LOCI-PSF method (Maire et al. 2012) can be used to take advantage of simultaneous measurement of the speckle pattern over several spectral channels as done by an IFS in addition to standard ADI speckle suppression.

\section{References}

Jolissaint, L., Véran, J.-P., et al. 2004, SPIE, 5490, 151-163

Lafrenière, D., Marois, C., Doyon, R., \& Nadeau, D. 2007, ApJ, 660, 770-780

Maire, J., Gagné, J., Lafrenière, D., Doyon, R., Graham, J. R., Véran, J.-P., \& Poyneer, L. A. 2012, SPIE, Volume 8447, article id. 844760, 14 pp. (2012)

Marois, C., Doyon, R., Racine, R., \& Nadeau, D. 2000, PASP, v112, 91-96

Poyneer, L. A., Véran, J.-P., et al. 2006, SPIE, 6272

Poyneer, L. A. Macintosh, B., et al. 2007, JOSAA, 24, 2645

Véran, J.-P., Rigaut, F., et al. 1997, JOSAA, 14, 11, 3057-3069 